\title{
An Iterative Method for Solving the Generalized System of Relaxed Cocoercive Quasivariational Inclusions and Fixed Point Problems of an Infinite Family of Strictly Pseudocontractive Mappings
}

\author{
Pattanapong Tianchai $^{\mathbf{1}}$ and Rabian Wangkeeree ${ }^{\mathbf{2}}$ \\ ${ }^{1}$ Faculty of Science, Maejo University, Chiangmai 50290, Thailand \\ ${ }^{2}$ Department of Mathematics, Faculty of Science, Naresuan University, Phisanulok 65000, Thailand \\ Correspondence should be addressed to Pattanapong Tianchai, pattana@mju.ac.th
}

Received 7 April 2010; Revised 27 July 2010; Accepted 18 September 2010

Academic Editor: Martin Bohner

Copyright (C) 2010 P. Tianchai and R. Wangkeeree. This is an open access article distributed under the Creative Commons Attribution License, which permits unrestricted use, distribution, and reproduction in any medium, provided the original work is properly cited.

We introduce an iterative scheme by the viscosity approximation to find the set of solutions of the generalized system of relaxed cocoercive quasivariational inclusions and the set of common fixed points of an infinite family of strictly pseudocontractive mappings problems in Hilbert spaces. We suggest and analyze an iterative scheme under some appropriate conditions imposed on the parameters; we prove that another strong convergence theorem for the above two sets is obtained. The results presented in this paper improve and extend the main results of $\mathrm{Li}$ and $\mathrm{Wu}(2010)$ and many others.

\section{Introduction and Preliminaries}

Let $H$ be a real Hilbert space with inner product and norm being denoted by $\langle\cdot, \cdot\rangle$ and $\|\cdot\|$, respectively, and let $C$ be a nonempty closed convex subset of $H$. Recall that $P_{C}$ is the metric projection of $H$ onto $C$; that is, for each $x \in H$ there exists the unique point in $P_{C} x \in C$ such that

$$
\left\|x-P_{C} x\right\|=\min _{y \in C}\|x-y\|
$$

A mapping $T: C \rightarrow C$ is called nonexpansive if

$$
\|T x-T y\| \leq\|x-y\|, \quad \forall x, y \in C
$$


and the mapping $f: C \rightarrow C$ is called a contraction if there exists a constant $\alpha \in(0,1)$ such that

$$
\|f(x)-f(y)\| \leq \alpha\|x-y\|, \quad \forall x, y \in C .
$$

A point $x \in C$ is a fixed point of $T$ provided $T x=x$. We denote by $F(T)$ the set of fixed points of $T$; that is, $F(T)=\{x \in C: T x=x\}$. If $C \subset H$ is bounded, closed and convex and $T$ is a nonexpansive mappings of $C$ into itself, then $F(T)$ is nonempty (see [1]). Recall that a mapping $A: C \rightarrow H$ is said to be

(i) monotone if

$$
\langle A x-A y, x-y\rangle \geq 0, \quad \forall x, y \in C
$$

(ii) $k$-Lipschitz continuous if there exists a constant $k>0$ such that

$$
\|A x-A y\| \leq k\|x-y\|, \quad \forall x, y \in C
$$

if $k=1$, then $A$ is a nonexpansive,

(iii) pseudocontractive if

$$
\|A x-A y\|^{2} \leq\|x-y\|^{2}+\|(I-A) x-(I-A) y\|^{2}, \quad \forall x, y \in C,
$$

(iv) $k$-strictly pseudocontractive if there exists a constant $k \in[0,1)$ such that

$$
\|A x-A y\|^{2} \leq\|x-y\|^{2}+k\|(I-A) x-(I-A) y\|^{2}, \quad \forall x, y \in C,
$$

it is obvious that $A$ is a nonexpansive if and only if $A$ is a 0 -strictly pseudocontractive,

(v) $\alpha$-strongly monotone if there exists a constant $\alpha>0$ such that

$$
\langle A x-A y, x-y\rangle \geq \alpha\|x-y\|^{2}, \quad \forall x, y \in C,
$$

(vi) $\alpha$-inverse-strongly monotone (or $\alpha$-cocoercive) if there exists a constant $\alpha>0$ such that

$$
\langle A x-A y, x-y\rangle \geq \alpha\|A x-A y\|^{2}, \quad \forall x, y \in C,
$$

if $\alpha=1$, then $A$ is called that firmly nonexpansive; it is obvious that any $\alpha$-inversestrongly monotone mapping $A$ is monotone and ( $1 / \alpha)$-Lipschitz continuous,

(vii) relaxed $\alpha$-cocoercive if there exists a constant $\alpha>0$ such that

$$
\langle A x-A y, x-y\rangle \geq(-\alpha)\|A x-A y\|^{2}, \quad \forall x, y \in C,
$$


(viii) relaxed $(\alpha, r)$-cocoercive if there exists two constants $\alpha, r>0$ such that

$$
\langle A x-A y, x-y\rangle \geq(-\alpha)\|A x-A y\|^{2}+r\|x-y\|^{2}, \quad \forall x, y \in C
$$

it is obvious that any the $r$-strongly monotonicity implies to the relaxed $(\alpha, r)$ cocoercivity.

Recall that a set-valued mapping $M: H \rightarrow 2^{H}$ is called monotone if for all $x, y \in$ $H, f \in M x$ and $g \in M y$ imply $\langle x-y, f-g\rangle \geq 0$. A monotone mapping $M: H \rightarrow 2^{H}$ is maximal if the graph of $G(M)$ of $M$ is not properly contained in the graph of any other monotone mappings.

The existence common fixed points for a finite family of nonexpansive mappings has been considered by many authers (see [2-5] and the references therein).

In this paper, we study the mapping $W_{n}$ defined by

$$
\begin{aligned}
U_{n, n+1} & =I, \\
U_{n, n} & =\mu_{n} S_{n} U_{n, n+1}+\left(1-\mu_{n}\right) I, \\
U_{n, n-1} & =\mu_{n-1} S_{n-1} U_{n, n}+\left(1-\mu_{n-1}\right) I, \\
& \vdots \\
U_{n, k} & =\mu_{k} S_{k} U_{n, k+1}+\left(1-\mu_{k}\right) I, \\
U_{n, k-1} & =\mu_{k-1} S_{k-1} U_{n, k}+\left(1-\mu_{k-1}\right) I, \\
& \vdots \\
U_{n, 2} & =\mu_{2} S_{2} U_{n, 3}+\left(1-\mu_{2}\right) I, \\
W_{n} & =U_{n, 1}=\mu_{1} S_{1} U_{n, 2}+\left(1-\mu_{1}\right) I,
\end{aligned}
$$

where $\left\{\mu_{i}\right\}$ is nonnegative real sequence in $(0,1)$, for all $i \in \mathbb{N}, S_{1}, S_{2}, \ldots$ form a family of infinitely nonexpansive mappings of $C$ into itself. It is obvious that $W_{n}$ is nonexpansive from $C$ into itself, such a mapping $W_{n}$ is called a $W$-mapping generated by $S_{1}, S_{2}, \ldots, S_{n}$ and $\mu_{1}, \mu_{2}, \ldots, \mu_{n}$.

A typical problem is to minimize a quadratic function over the set of fixed points of a nonexpansive mapping in a real Hilbert space $H$ :

$$
\min _{x \in C}\left\{\frac{1}{2}\langle A x, x\rangle-\langle x, b\rangle\right\}
$$

where $A$ is a bounded linear operator on $H, C$ is the fixed point set of a nonexpansive mapping $S$ on $H$ and $b$ is a given point in $H$. Recall that $A$ be a strongly positive bounded linear operator on $H$ if there exists a constant $\bar{\gamma}>0$ such that

$$
\langle A x, x\rangle \geq \bar{\gamma}\|x\|^{2}, \quad \forall x \in H .
$$


Marino and $\mathrm{Xu}$ [6] introduced the following general iterative scheme based on the viscosity approximation method introduced by Moudafi [7]:

$$
x_{n+1}=\alpha_{n} \gamma f\left(x_{n}\right)+\left(I-\alpha_{n} A\right) S x_{n}, \quad \forall n \in \mathbb{N},
$$

where $A$ is a strongly positive bounded linear operator on $H, f$ is a contraction on $H$ and $S$ is a nonexpansive on $H$. They proved that under those conditions are corrected, if $F(S) \neq \emptyset$, then the sequence $\left\{x_{n}\right\}$ generated by (1.15) converges strongly to the unique solution $z$ of the variational inequality

$$
\langle(A-\gamma f) z, x-z\rangle \geq 0, \quad \forall x \in F(S)
$$

which is the optimality condition for the minimization problem

$$
\min _{x \in F(S)}\left\{\frac{1}{2}\langle A x, x\rangle-h(x)\right\}
$$

where $h$ is a potential function for $\gamma f$ (i.e., $h^{\prime}(x)=\gamma f(x)$ for $x \in H$ ).

The so-called the system of generalized quasivariational inclusions problem is to find $\left(x^{*}, y^{*}\right) \in H \times H$ such that

$$
\begin{aligned}
& 0 \in x^{*}-y^{*}+\lambda_{1}\left(\left(B_{1}+C_{1}\right) y^{*}+M_{1} x^{*}\right), \\
& 0 \in y^{*}-x^{*}+\lambda_{2}\left(\left(B_{2}+C_{2}\right) x^{*}+M_{2} y^{*}\right),
\end{aligned}
$$

where $B_{i}, C_{i}: H \rightarrow H, M_{i}: H \rightarrow 2^{H}$ are nonlinear mappings and $\lambda_{i}>0$ for each $i=1,2$. As special cases of problem (1.18), we have the following.

(1) If $B_{1}=B_{2}=B, C_{1}=C_{2}=C$ and $M_{1}=M_{2}=M$, then problem (1.18) is reduced to find $\left(x^{*}, y^{*}\right) \in H \times H$ such that

$$
\begin{aligned}
& 0 \in x^{*}-y^{*}+\lambda_{1}\left((B+C) y^{*}+M x^{*}\right), \\
& 0 \in y^{*}-x^{*}+\lambda_{2}\left((B+C) x^{*}+M y^{*}\right) .
\end{aligned}
$$

(2) If $C_{1}=C_{2}=0$, then problem (1.18) is reduced to find $\left(x^{*}, y^{*}\right) \in H \times H$ such that

$$
\begin{aligned}
& 0 \in x^{*}-y^{*}+\lambda_{1}\left(B_{1} y^{*}+M_{1} x^{*}\right), \\
& 0 \in y^{*}-x^{*}+\lambda_{2}\left(B_{2} x^{*}+M_{2} y^{*}\right),
\end{aligned}
$$

which called that the system of quasivariational inclusions problem. 
(3) If $B_{1}=B_{2}=B$ and $M_{1}=M_{2}=M$, then problem (1.20) is reduced to find $\left(x^{*}, y^{*}\right) \in$ $H \times H$ such that

$$
\begin{aligned}
& 0 \in x^{*}-y^{*}+\lambda_{1}\left(B y^{*}+M x^{*}\right), \\
& 0 \in y^{*}-x^{*}+\lambda_{2}\left(B x^{*}+M y^{*}\right) .
\end{aligned}
$$

(4) If $x^{*}=y^{*}$, then problem (1.21) is reduced to find $x^{*} \in H$ such that

$$
0 \in B x^{*}+M x^{*}
$$

We denote by $\operatorname{VI}(H, B, M)$ the set of solutions of variational inclusion of the problem (1.22).

(5) If $M=\partial \phi: H \rightarrow 2^{H}$, where $\phi: H \rightarrow \mathbb{R} \cup\{+\infty\}$ is a proper convex lower semicontinuous function and $\partial \phi$ is the subdifferential of $\phi$, then problem (1.22) is equivalent to find $x^{*} \in H$ such that

$$
\left\langle B x^{*}, v-x^{*}\right\rangle+\phi(v)-\phi\left(x^{*}\right) \geq 0, \quad \forall v \in H,
$$

which is said to be the mixed quasivariational inequality (see, e.g., $[8,9]$ for more details).

(6) If $\phi$ is the indicator function of $C$, then problem (1.23) is equivalent to the classical variational inequality problem, denoted by $\operatorname{VI}(C, B)$, to find $x^{*} \in C$ such that

$$
\left\langle B x^{*}, v-x^{*}\right\rangle \geq 0, \quad \forall v \in C .
$$

Iiduka and Takahashi [10] introduced iterative scheme for finding a common element of the set of fixed points of a nonexpansive mapping and the set of solutions of the variational inequality (1.24) as the following theorem.

Theorem IT. Let $C$ be a closed convex subset of a real Hilbert space $H$. Let $B$ be an $\alpha$-inversestrongly monotone mapping of $C$ into $H$ and let $S$ be a nonexpansive mapping of $C$ into itself such that $F(S) \cap V I(C, B) \neq \emptyset$. Suppose that $x_{1}=x \in C$ and $\left\{x_{n}\right\}$ is the sequence defined by

$$
x_{n+1}=\alpha_{n} x+\left(1-\alpha_{n}\right) S P_{C}\left(x_{n}-\lambda_{n} B x_{n}\right), \quad \forall n \in \mathbb{N},
$$

where $\left\{\alpha_{n}\right\} \subset[0,1)$ and $\left\{\lambda_{n}\right\} \subset[a, b]$ for some $a, b \in(0,2 \alpha)$ satisfying the following conditions:

(C1) $\lim _{n \rightarrow \infty} \alpha_{n}=0$ and $\sum_{n=1}^{\infty} \alpha_{n}=\infty$;

(C2) $\sum_{n=1}^{\infty}\left|\alpha_{n+1}-\alpha_{n}\right|<\infty$ and $\sum_{n=1}^{\infty}\left|\lambda_{n+1}-\lambda_{n}\right|<\infty$.

Then $\left\{x_{n}\right\}$ converges strongly to $P_{F(S) \cap V I(C, B)} x$.

Definition 1.1 (see [11]). Let $M: H \rightarrow 2^{H}$ be a multivalued maximal monotone mapping. Then the single-valued mapping $J_{M, \lambda}: H \rightarrow H$ defined by $J_{M, \lambda}(u)=(I+\lambda M)^{-1}(u)$, for all $u \in H$, is called the resolvent operator associated with $M$, where $\lambda$ is any positive number and $I$ is the identity mapping. 
Recently, Zhang et al. [11] considered the problem (1.22). To be more precise, they proved the following theorem.

Theorem ZLC. Let $H$ be a real Hilbert space, $B: H \rightarrow H$ be an $\alpha$-inverse-strongly monotone mapping, $M: H \rightarrow 2^{H}$ be a maximal monotone mapping, and $S: H \rightarrow H$ be a nonexpansive mapping. Suppose that the set $F(S) \cap V I(H, B, M) \neq \emptyset$, where $V I(H, B, M)$ is the set of solutions of variational inclusion (1.22). Suppose that $x_{1}=x \in H$ and $\left\{x_{n}\right\}$ is the sequence defined by

$$
\begin{gathered}
y_{n}=J_{M, \lambda}\left(x_{n}-\lambda B x_{n}\right), \\
x_{n+1}=\alpha_{n} x+\left(1-\alpha_{n}\right) S y_{n},
\end{gathered}
$$

for all $n \in \mathbb{N}$, where $\lambda \in(0,2 \alpha)$ and $\left\{\alpha_{n}\right\} \subset[0,1]$ satisfying the following conditions:

(C1) $\lim _{n \rightarrow \infty} \alpha_{n}=0$ and $\sum_{n=1}^{\infty} \alpha_{n}=\infty$;

(C2) $\sum_{n=1}^{\infty}\left|\alpha_{n+1}-\alpha_{n}\right|<\infty$.

Then $\left\{x_{n}\right\}$ converges strongly to $P_{F(S) \cap V I(H, B, M)} x$.

Very recently, Li and $\mathrm{Wu}[12]$ introduced an iterative scheme:

$$
\begin{gathered}
y_{n}=J_{M, \lambda}\left(x_{n}-\lambda B x_{n}\right), \\
x_{n+1}=\alpha_{n} \gamma f\left(x_{n}\right)+\beta_{n} x_{n}+\left(\left(1-\beta_{n}\right) I-\alpha_{n} A\right)\left(\mu S_{k} x_{n}+(1-\mu) y_{n}\right),
\end{gathered}
$$

for all $n \in \mathbb{N}$, where $x_{1} \in H, A$ is a strongly positive bounded linear operator on $H, f$ is a contraction on $H$ and $S_{k}$ is a mapping on $H$ defined by $S_{k} x=k x+(1-k) S x$ such that $S$ is a $k$-strictly pseudocontractive mapping on $H$ with a fixed point. They proved that under missing condition of $\mu$, it should be $0<\mu<1$ by those Lemma 1.6, others are corrected, if $\Omega=F(S) \cap \mathrm{VI}(H, B, M) \neq \emptyset$, then the sequence $\left\{x_{n}\right\}$ generated by (1.27) converges strongly to $z=P_{\Omega}(I-A+\gamma f) z$ of the variational inequality

$$
\langle(A-\gamma f) z, x-z\rangle \geq 0, \quad \forall x \in \Omega,
$$

which is the optimality condition for the minimization problem:

$$
\min _{x \in \Omega}\left\{\frac{1}{2}\langle A x, x\rangle-h(x)\right\}
$$

where $h$ is a potential function for $\gamma f$.

Inspired and motivated by the works mentioned above, in this paper, we introduce an iterative scheme (2.2) below by the viscosity approximation to find the set of solutions of the generalized system of relaxed cocoercive quasivariational inclusions and the set of common fixed points of an infinite family of strictly pseudocontractive mappings problems in Hilbert spaces. We suggest and analyze an iterative scheme under some appropriate conditions imposed on the parameters, we prove that another strong convergence theorem for the above two sets is obtained. The results presented in this paper improve and extend the main results of $\mathrm{Li}$ and $\mathrm{Wu}[12]$ and many others. 
We collect the following lemmas which be used in the proof for the main results in the next section.

Lemma 1.2 (see [6]). Let $H$ be a Hilbert space, $C$ be a nonempty closed convex subset of $H, f$ : $H \rightarrow H$ be a contraction with coefficient $0<\alpha<1$, and $A$ be a strongly positive linear bounded operator with coefficient $\bar{\gamma}>0$. Then,

(1) if $0<\gamma<\bar{\gamma} / \alpha$, then $\langle x-y,(A-\gamma f) x-(A-\gamma f) y\rangle \geq(\bar{\gamma}-\gamma \alpha)\|x-y\|^{2}, x, y \in H$;

(2) if $0<\rho \leq\|A\|^{-1}$, then $\|I-\rho A\| \leq 1-\rho \bar{\gamma}$.

Lemma 1.3 (see [13]). Let $\left\{x_{n}\right\}$ and $\left\{z_{n}\right\}$ be bounded sequences in a Banach space $E$ and $\left\{\beta_{n}\right\}$ be a sequence in $[0,1]$ which satisfies the following condition:

$$
0<\liminf _{n \rightarrow \infty} \beta_{n} \leq \limsup _{n \rightarrow \infty} \beta_{n}<1
$$

Suppose that

$$
x_{n+1}=\left(1-\beta_{n}\right) x_{n}+\beta_{n} z_{n}, \quad n \geq 1
$$

and $\lim \sup _{n \rightarrow \infty}\left(\left\|z_{n+1}-z_{n}\right\|-\left\|x_{n+1}-x_{n}\right\|\right) \leq 0$. Then $\lim _{n \rightarrow \infty}\left\|z_{n}-x_{n}\right\|=0$.

Lemma 1.4 (see [14]). Assume $\left\{a_{n}\right\}$ is a sequence of nonnegative real numbers such that

$$
a_{n+1} \leq\left(1-\eta_{n}\right) a_{n}+\delta_{n}, \quad n \geq 1
$$

where $\left\{\eta_{n}\right\}$ is a sequence in $(0,1)$ and $\left\{\delta_{n}\right\}$ is a sequence in $\mathbb{R}$ such that

(1) $\sum_{n=1}^{\infty} \eta_{n}=\infty$;

(2) $\lim \sup _{n \rightarrow \infty}\left(\delta_{n} / \eta_{n}\right) \leq 0$ or $\sum_{n=1}^{\infty}\left|\delta_{n}\right|<\infty$.

Then $\lim _{n \rightarrow \infty} a_{n}=0$.

Lemma 1.5 (see [15]). Let $C$ be a nonempty closed convex subset of a Hilbert space $H$, define mapping $W_{n}$ as (1.12), let $S_{i}: C \rightarrow C$ be a family of infinitely nonexpansive mappings with $\bigcap_{i=1}^{\infty} F\left(S_{i}\right) \neq \emptyset$, and let $\left\{\mu_{i}\right\}$ be a sequence such that $0<\mu_{i} \leq \mu<1$, for all $i \geq 1$. Then

(1) $W_{n}$ is nonexpansive and $F\left(W_{n}\right)=\bigcap_{i=1}^{\infty} F\left(S_{i}\right)$ for each $n \geq 1$;

(2) for each $x \in C$ and for each positive integer $k, \lim _{n \rightarrow \infty} U_{n, k} x$ exists;

(3) the mapping $W: C \rightarrow C$ define by

$$
W x:=\lim _{n \rightarrow \infty} W_{n} x=\lim _{n \rightarrow \infty} U_{n, 1} x, \quad x \in C,
$$

is a nonexpansive mapping satisfying $F(W)=\bigcap_{i=1}^{\infty} F\left(S_{i}\right)$ and it is called the $W$-mapping generated by $S_{1}, S_{2}, \ldots$ and $\mu_{1}, \mu_{2}, \ldots$

Lemma 1.6 (see [11]). The resolvent operator $J_{M, \lambda}$ associated with $M$ is single-valued and nonexpansive for all $\lambda>0$. 
Lemma 1.7 (see [11]). $u \in H$ is a solution of variational inclusion (1.22) if and only if $u=J_{M, \lambda}(u-$ $\lambda B u)$, for all $\lambda>0$, that is,

$$
\mathrm{VI}(H, B, M)=F\left(J_{M, \lambda}(I-\lambda B)\right), \quad \forall \lambda>0 .
$$

Lemma 1.8. For any $\left(x^{*}, y^{*}\right) \in H \times H$, where $y^{*}=J_{M_{2}, \lambda_{2}}\left(I-\lambda_{2}\left(B_{2}+C_{2}\right)\right) x^{*}$, we have $\left(x^{*}, y^{*}\right)$ is a solution of problem (1.18) if and only if $x^{*}$ is a fixed point of the mapping $D$ defined by

$$
D x=J_{M_{1}, \lambda_{1}}\left(\left(I-\lambda_{1}\left(B_{1}+C_{1}\right)\right) J_{M_{2}, \lambda_{2}}\left(I-\lambda_{2}\left(B_{2}+C_{2}\right)\right) x\right) \text {. }
$$

Proof. Observe from (1.18) that

$$
\begin{aligned}
& 0 \in x^{*}-y^{*}+\lambda_{1}\left(\left(B_{1}+C_{1}\right) y^{*}+M_{1} x^{*}\right), \\
& 0 \in y^{*}-x^{*}+\lambda_{2}\left(\left(B_{2}+C_{2}\right) x^{*}+M_{2} y^{*}\right) \\
& x^{*}=J_{M_{1}, \lambda_{1}}\left(I-\lambda_{1}\left(B_{1}+C_{1}\right)\right) y^{*}, \\
& y^{*}=J_{M_{2}, \lambda_{2}}\left(I-\lambda_{2}\left(B_{2}+C_{2}\right)\right) x^{*} \\
\Longleftrightarrow & D x^{*}=J_{M_{1}, \lambda_{1}}\left(\left(I-\lambda_{1}\left(B_{1}+C_{1}\right)\right) J_{M_{2,}, \lambda_{2}}\left(I-\lambda_{2}\left(B_{2}+C_{2}\right)\right) x^{*}\right)=x^{*} .
\end{aligned}
$$

Lemma 1.9 (see [16]). Let $C$ be a closed convex subset of a strictly convex Banach space E. Let $S$ and $T$ be two nonexpansive mappings on $C$. Suppose that $F(S) \cap F(T)$ is nonempty. Then a mapping $R$ on $C$ defined by $R x=a S x+(1-a) T x$, where $a \in(0,1)$, for $x \in C$ is well defined and nonexpansive and $F(R)=F(S) \cap F(T)$ holds.

Lemma 1.10 (see [17]). Let $H$ be a real Hilbert space, let $C$ be a nonempty closed convex subset of $H$, and let $S: C \rightarrow C$ be a nonexpansive mapping. Then $I-S$ is demiclosed at zero.

Lemma 1.11 (see [18]). Let $C$ be a nonempty closed convex subset of a real Hilbert space $H$ and $T: C \rightarrow C$ be a $k$-strict pseudocontraction. Define $S: C \rightarrow H$ by $S x=\alpha x+(1-\alpha) T x$ for each $x \in C$. Then, as $\alpha \in[k, 1), S$ is a nonexpansive such that $F(S)=F(T)$.

\section{Main Results}

Let $H$ be a real Hilbert space, $M_{i}: H \rightarrow 2^{H}$ be a maximal monotone mapping, $B_{i}: H \rightarrow H$ be a relaxed $\left(\alpha_{i}, r_{i}\right)$-cocoercive and $\xi_{i}$-Lipschitz continuous mappings, respectively, $C_{i}: H \rightarrow$ $H$ be a relaxed $\left(\bar{\alpha}_{i}, \bar{r}_{i}\right)$-cocoercive and $\bar{\xi}_{i}$-Lipschitz continuous mappings, respectively, for each $i=1$, 2. Let $A: H \rightarrow H$ be a strongly positive linear bounded self-adjoint operator mapping with coefficient $\bar{\delta} \in(0,1]$ such that $\|A\| \leq 1$ and $f: H \rightarrow H$ be a contraction mapping with coefficient $\delta \in(0,1)$. Let $\left\{T_{n}: H \rightarrow H\right\}$ be a family of $k_{n}$-strictly pseudocontractive mappings with a fixed point such that $k_{n} \in[0,1)$ for all $n \in \mathbb{N}$. Define $S_{n} x=\delta_{n} x+\left(1-\delta_{n}\right) T_{n} x$, where $\delta_{n} \in\left[k_{n}, 1\right)$, for all $n \in \mathbb{N}$, and let $W_{n}: H \rightarrow H$ be a $W$-mapping generated by $\left\{S_{n}\right\}$ and $\left\{\mu_{n}\right\}$ such that $\left\{\mu_{n}\right\} \subset(0, \mu]$, for some $\mu \in(0,1)$. 
Define sequence of mappings $\left\{P_{n}: H \rightarrow H\right\}$ and mapping $Q: H \rightarrow H$ as follows:

$$
\begin{gathered}
P_{n} x=\alpha_{n} \gamma f\left(W_{n} x\right)+\beta_{n} x+\left(\left(1-\beta_{n}\right) I-\alpha_{n} A\right)\left(\gamma_{n} W_{n} x+\left(1-\gamma_{n}\right) Q x\right), \\
Q x=J_{M_{1}, \lambda_{1}}\left(\left(I-\lambda_{1}\left(B_{1}+C_{1}\right)\right) J_{M_{2}, \lambda_{2}}\left(I-\lambda_{2}\left(B_{2}+C_{2}\right)\right) x\right),
\end{gathered}
$$

for all $n \in \mathbb{N}$, where $\left\{\alpha_{n}\right\},\left\{\beta_{n}\right\},\left\{\gamma_{n}\right\} \subset\left(0,\|A\|^{-1}\right), \lambda_{i} \in\left(0, \epsilon_{i}\right], \epsilon_{i}=\min \left\{2\left(r_{i}-\alpha_{i} \xi_{i}^{2}\right) / \xi_{i}\left(\xi_{i}+\right.\right.$ $\left.\left.\bar{\xi}_{i}\right), 2\left(\bar{r}_{i}-\bar{\alpha}_{i} \bar{\xi}_{i}^{2}\right) / \bar{\xi}_{i}\left(\xi_{i}+\bar{\xi}_{i}\right)\right\}, r_{i}>\alpha_{i} \xi_{i}^{2}, \bar{r}_{i}>\bar{\alpha}_{i} \bar{\xi}_{i}^{2}$, for each $i=1,2$ and $0<\gamma<\bar{\delta} / \delta$.

Under some appropriate imposed on the parameters $\left\{\alpha_{n}\right\}$ and $\left\{\beta_{n}\right\}$, we also know that $\left\|\left(1-\beta_{n}\right) I-\alpha_{n} A\right\| \leq 1-\beta_{n}-\alpha_{n} \bar{\delta}$, and, we also have that $W_{n}, J_{M_{i}, \lambda_{i}}$ and $I-\lambda_{i}\left(B_{i}+C_{i}\right)$ are nonexpansive for each $i=1,2$ (see argument in the proof of Theorem 2.1 below). Observe that $Q$ is a nonexpansive, and so $P_{n}$ is a contraction with coefficient $1-(\bar{\delta}-\gamma \delta) \alpha_{n}$. Therefore, by Banach contraction principle guarantees that $P_{n}$ has a unique fixed point in $H$.

By the idea above, we obtain an iteration scheme by the viscosity approximation for solving the generalized system of relaxed cocoercive quasivariational inclusions and fixed point problems of an infinite family of strictly pseudocontractive mappings as the following theorem.

Theorem 2.1. Let $H$ be a real Hilbert space, $M_{i}: H \rightarrow 2^{H}$ be a maximal monotone mapping, $B_{i}: H \rightarrow H$ be a relaxed $\left(\alpha_{i}, r_{i}\right)$-cocoercive and $\xi_{i}$-Lipschitz continuous mappings, respectively, $C_{i}: H \rightarrow H$ be a relaxed $\left(\bar{\alpha}_{i}, \bar{r}_{i}\right)$-cocoercive and $\bar{\xi}_{i}$-Lipschitz continuous mappings, respectively, for each $i=1$,2. Let $A: H \rightarrow H$ be a strongly positive linear bounded self-adjoint operator mapping with coefficient $\bar{\delta} \in(0,1]$ such that $\|A\| \leq 1$ and $f: H \rightarrow H$ be a contraction mapping with coefficient $\delta \in(0,1)$. Let $\left\{T_{n}: H \rightarrow H\right\}$ be a family of $k_{n}$-strictly pseudocontractive mappings with a fixed point such that $k_{n} \in[0,1)$ for all $n \in \mathbb{N}$. Define $S_{n} x=\delta_{n} x+\left(1-\delta_{n}\right) T_{n} x$, where $\delta_{n} \in\left[k_{n}, 1\right)$, for all $n \in \mathbb{N}$, and let $W_{n}: H \rightarrow H$ be $a W$-mapping generated by $\left\{S_{n}\right\}$ and $\left\{\mu_{n}\right\}$ such that $\left\{\mu_{n}\right\} \subset(0, \mu]$, for some $\mu \in(0,1)$. Assume that $\Omega:=\bigcap_{n=1}^{\infty} F\left(T_{n}\right) \cap F(D) \neq \emptyset$ and $0<\gamma<\bar{\delta} / \delta$ where $D: H \rightarrow H$ defined by $D x=J_{M_{1}, \lambda_{1}}\left(\left(I-\lambda_{1}\left(B_{1}+C_{1}\right)\right) J_{M_{2}, \lambda_{2}}\left(I-\lambda_{2}\left(B_{2}+C_{2}\right)\right) x\right)$. For $x_{1} \in H$, suppose that $\left\{x_{n}\right\}$ be generated iteratively by

$$
\begin{gathered}
z_{n}=J_{M_{2}, \lambda_{2}}\left(x_{n}-\lambda_{2}\left(B_{2}+C_{2}\right) x_{n}\right), \\
y_{n}=J_{M_{1}, \lambda_{1}}\left(z_{n}-\lambda_{1}\left(B_{1}+C_{1}\right) z_{n}\right), \\
x_{n+1}=\alpha_{n} \gamma f\left(W_{n} x_{n}\right)+\beta_{n} x_{n}+\left(\left(1-\beta_{n}\right) I-\alpha_{n} A\right)\left(\gamma_{n} W_{n} x_{n}+\left(1-\gamma_{n}\right) y_{n}\right),
\end{gathered}
$$

for all $n \in \mathbb{N}$, where $\left\{\alpha_{n}\right\},\left\{\beta_{n}\right\},\left\{\gamma_{n}\right\} \subset(0,1), \lambda_{i} \in\left(0, \epsilon_{i}\right], \epsilon_{i}=\min \left\{2\left(r_{i}-\alpha_{i} \xi_{i}^{2}\right) / \xi_{i}\left(\xi_{i}+\bar{\xi}_{i}\right), 2\left(\bar{r}_{i}-\right.\right.$ $\left.\left.\bar{\alpha}_{i} \bar{\xi}_{i}^{2}\right) / \bar{\xi}_{i}\left(\xi_{i}+\bar{\xi}_{i}\right)\right\}, r_{i}>\alpha_{i} \xi_{i}^{2}, \bar{r}_{i}>\bar{\alpha}_{i} \bar{\xi}_{i}^{2}$, for each $i=1,2$, satisfying the following conditions:

(C1) $\lim _{n \rightarrow \infty} \alpha_{n}=\lim _{n \rightarrow \infty}\left|\gamma_{n+1}-\gamma_{n}\right|=0$;

(C2) $0<\liminf _{n \rightarrow \infty} \beta_{n} \leq \lim \sup _{n \rightarrow \infty} \beta_{n}<1$;

(C3) $\sum_{n=1}^{\infty} \alpha_{n}=\infty$ and $0<\lim _{n \rightarrow \infty} \gamma_{n}<1$. 
Then the sequence $\left\{x_{n}\right\}$ converges strongly to $w \in \Omega$ where $w=P_{\Omega}(I-A+\gamma f)$ w is a unique solution of the variational inequality

$$
\langle(A-\gamma f) w, y-w\rangle \geq 0, \quad \forall y \in \Omega,
$$

which is the optimality condition for the minimization problem

$$
\min _{x \in \Omega}\left\{\frac{1}{2}\langle A x, x\rangle-h(x)\right\}
$$

where $h$ is a potential function for $\gamma f$ (i.e., $h^{\prime}(x)=\gamma f(x)$ for $\left.x \in H\right)$ and $\left(w, J_{M_{2}, \lambda_{2}}\left(I-\lambda_{2}\left(B_{2}+\right.\right.\right.$ $\left.\left.C_{2}\right)\right) w$ ) is a solution of problem (1.18).

Proof. From (C1) and (C2), we have $\alpha_{n} \rightarrow 0$ and lim $\sup _{n \rightarrow \infty} \beta_{n}<1$. Thus, we may assume without loss of generality that $\alpha_{n} \leq\left(1-\beta_{n}\right)\|A\|^{-1}$ for all $n \in \mathbb{N}$. For each $i=1,2$, since

$$
0<\lambda_{i} \leq \epsilon_{i} \leq \frac{2\left(r_{i}-\alpha_{i} \xi_{i}^{2}\right)}{\xi_{i}\left(\xi_{i}+\bar{\xi}_{i}\right)}, \quad 0<\lambda_{i} \leq \epsilon_{i} \leq \frac{2\left(\bar{r}_{i}-\bar{\alpha}_{i} \bar{\xi}_{i}^{2}\right)}{\bar{\xi}_{i}\left(\xi_{i}+\bar{\xi}_{i}\right)}
$$

where $r_{i}>\alpha_{i} \xi_{i}^{2}$ and $\bar{r}_{i}>\bar{\alpha}_{i} \bar{\xi}_{i}^{2}$, we have

$$
\lambda_{i}^{2} \xi_{i}\left(\xi_{i}+\bar{\xi}_{i}\right)+2 \lambda_{i} \alpha_{i} \xi_{i}^{2}-2 \lambda_{i} r_{i} \leq 0, \quad \lambda_{i}^{2} \bar{\xi}_{i}\left(\xi_{i}+\bar{\xi}_{i}\right)+2 \lambda_{i} \bar{\alpha}_{i} \bar{\xi}_{i}^{2}-2 \lambda_{i} \bar{r}_{i} \leq 0
$$

For any $x, y \in H$, it follows by the relaxed $\left(\alpha_{i}, r_{i}\right)$-cocoercivity and $\xi_{i}$-Lipschitz continuity of $B_{i}$ and the relaxed $\left(\bar{\alpha}_{i}, \bar{r}_{i}\right)$-cocoercivity and $\bar{\xi}_{i}$-Lipschitz continuity of $C_{i}$ that

$$
\begin{aligned}
\|(I- & \left.\lambda_{i}\left(B_{i}+C_{i}\right)\right) x-\left(I-\lambda_{i}\left(B_{i}+C_{i}\right)\right) y \|^{2} \\
= & \left\|(x-y)-\lambda_{i}\left(\left(B_{i}+C_{i}\right) x-\left(B_{i}+C_{i}\right) y\right)\right\|^{2} \\
= & \|x-y\|^{2}-2 \lambda_{i}\left\langle x-y,\left(B_{i}+C_{i}\right) x-\left(B_{i}+C_{i}\right) y\right\rangle+\lambda_{i}^{2}\left\|\left(B_{i}+C_{i}\right) x-\left(B_{i}+C_{i}\right) y\right\|^{2} \\
= & \|x-y\|^{2}-2 \lambda_{i}\left\langle x-y, B_{i} x-B_{i} y\right\rangle-2 \lambda_{i}\left\langle x-y, C_{i} x-C_{i} y\right\rangle \\
& +\lambda_{i}^{2}\left\|\left(B_{i} x-B_{i} y\right)+\left(C_{i} x-C_{i} y\right)\right\|^{2}
\end{aligned}
$$


International Journal of Mathematics and Mathematical Sciences

$$
\begin{aligned}
\leq & \|x-y\|^{2}-2 \lambda_{i}\left(-\alpha_{i}\left\|B_{i} x-B_{i} y\right\|^{2}+r_{i}\|x-y\|^{2}\right)-2 \lambda_{i}\left(-\bar{\alpha}_{i}\left\|C_{i} x-C_{i} y\right\|^{2}+\bar{r}_{i}\|x-y\|^{2}\right) \\
& +\lambda_{i}^{2}\left(\left\|B_{i} x-B_{i} y\right\|+\left\|C_{i} x-C_{i} y\right\|\right)^{2} \\
\leq & \|x-y\|^{2}-2 \lambda_{i}\left(-\alpha_{i} \xi_{i}^{2}\|x-y\|^{2}+r_{i}\|x-y\|^{2}\right)-2 \lambda_{i}\left(-\bar{\alpha}_{i} \bar{\xi}_{i}^{2}\|x-y\|^{2}+\bar{r}_{i}\|x-y\|^{2}\right) \\
& +\lambda_{i}^{2}\left(\xi_{i}\|x-y\|+\bar{\xi}_{i}\|x-y\|\right)^{2} \\
= & \left(1+\left(\lambda_{i}^{2} \xi_{i}\left(\xi_{i}+\bar{\xi}_{i}\right)+2 \lambda_{i} \alpha_{i} \xi_{i}^{2}-2 \lambda_{i} r_{i}\right)+\left(\lambda_{i}^{2} \bar{\xi}_{i}\left(\xi_{i}+\bar{\xi}_{i}\right)+2 \lambda_{i} \bar{\alpha}_{i} \bar{\xi}_{i}^{2}-2 \lambda_{i} \bar{r}_{i}\right)\right)\|x-y\|^{2} \\
\leq & \|x-y\|^{2},
\end{aligned}
$$

which implies that $I-\lambda_{i}\left(B_{i}+C_{i}\right)$ is a nonexpansive. Since, $A$ is a linear bounded self-adjoint operator, we have

$$
\|A\|=\sup \{|\langle A x, x\rangle|: x \in E,\|x\|=1\} .
$$

Observe that

$$
\begin{aligned}
\left\langle\left(\left(1-\beta_{n}\right) I-\alpha_{n} A\right) x, x\right\rangle & =\left(1-\beta_{n}\right)\langle x, x\rangle-\alpha_{n}\langle A x, x\rangle \\
& \geq\left(1-\beta_{n}\right)-\alpha_{n}\|A\| \\
& \geq 0 .
\end{aligned}
$$

Therefore, we obtain $\left(1-\beta_{n}\right) I-\alpha_{n} A$ is positive. Thus, by the strong positively of $A$, we get

$$
\begin{aligned}
\left\|\left(1-\beta_{n}\right) I-\alpha_{n} A\right\| & =\sup \left\{\left\langle\left(\left(1-\beta_{n}\right) I-\alpha_{n} A\right) x, x\right\rangle: x \in E,\|x\|=1\right\} \\
& =\sup \left\{\left(1-\beta_{n}\right)\langle x, x\rangle-\alpha_{n}\langle A x, x\rangle: x \in E,\|x\|=1\right\} \\
& \leq 1-\beta_{n}-\alpha_{n} \bar{\delta} .
\end{aligned}
$$

Pick $x^{*} \in \Omega$. Then, we have

$$
x^{*}=D x^{*}=J_{M_{1}, \lambda_{1}}\left(\left(I-\lambda_{1}\left(B_{1}+C_{1}\right)\right) J_{M_{2}, \lambda_{2}}\left(I-\lambda_{2}\left(B_{2}+C_{2}\right)\right) x^{*}\right) .
$$


And, let $y^{*}=J_{M_{2}, \lambda_{2}}\left(x^{*}-\lambda_{2}\left(B_{2}+C_{2}\right) x^{*}\right)$. Therefore, from (2.11), we have

$$
x^{*}=J_{M_{1}, \lambda_{1}}\left(y^{*}-\lambda_{1}\left(B_{1}+C_{1}\right) y^{*}\right) .
$$

By the nonexpansivity of $J_{M_{i}, \lambda_{i}}$ and $I-\lambda_{i}\left(B_{i}+C_{i}\right)$, we have

$$
\begin{aligned}
\left\|z_{n}-y^{*}\right\| & =\left\|J_{M_{2,} \lambda_{2}}\left(x_{n}-\lambda_{2}\left(B_{2}+C_{2}\right) x_{n}\right)-J_{M_{2}, \lambda_{2}}\left(x^{*}-\lambda_{2}\left(B_{2}+C_{2}\right) x^{*}\right)\right\| \\
& \leq\left\|\left(x_{n}-\lambda_{2}\left(B_{2}+C_{2}\right) x_{n}\right)-\left(x^{*}-\lambda_{2}\left(B_{2}+C_{2}\right) x^{*}\right)\right\| \\
& \leq\left\|x_{n}-x^{*}\right\| .
\end{aligned}
$$

And, we have

$$
\begin{aligned}
\left\|y_{n}-x^{*}\right\| & =\left\|J_{M_{1}, \lambda_{1}}\left(z_{n}-\lambda_{1}\left(B_{1}+C_{1}\right) z_{n}\right)-J_{M_{1}, \lambda_{1}}\left(y^{*}-\lambda_{1}\left(B_{1}+C_{1}\right) y^{*}\right)\right\| \\
& \leq\left\|\left(z_{n}-\lambda_{1}\left(B_{1}+C_{1}\right) z_{n}\right)-\left(y^{*}-\lambda_{1}\left(B_{1}+C_{1}\right) y^{*}\right)\right\| \\
& \leq\left\|z_{n}-y^{*}\right\| .
\end{aligned}
$$

Therefore, by (2.13) and (2.14), we have

$$
\left\|y_{n}-x^{*}\right\| \leq\left\|z_{n}-y^{*}\right\| \leq\left\|x_{n}-x^{*}\right\|
$$

Let $t_{n}=\gamma_{n} W_{n} x_{n}+\left(1-\gamma_{n}\right) y_{n}$. Since $S_{n} x=\delta_{n} x+\left(1-\delta_{n}\right) T_{n} x$, where $\delta_{n} \in\left[k_{n}, 1\right)$ and $\left\{T_{n}\right\}$ be a family of $k_{n}$-strict pseudocontraction. By Lemma 1.11, we have $S_{n}$ is a nonexpansive and $F\left(S_{n}\right)=F\left(T_{n}\right)$. Therefore, by Lemma 1.5(1), we get $F\left(W_{n}\right)=\bigcap_{i=1}^{\infty} F\left(S_{i}\right)=\bigcap_{i=1}^{\infty} F\left(T_{i}\right)$, which implies that $W_{n} x^{*}=x^{*}$. It follows by (2.15) and the nonexpansivity of $W_{n}$ that

$$
\begin{aligned}
\left\|t_{n}-x^{*}\right\| & =\left\|\gamma_{n} W_{n} x_{n}+\left(1-\gamma_{n}\right) y_{n}-x^{*}\right\| \\
& =\left\|\gamma_{n}\left(W_{n} x_{n}-x^{*}\right)+\left(1-\gamma_{n}\right)\left(y_{n}-x^{*}\right)\right\| \\
& \leq \gamma_{n}\left\|x_{n}-x^{*}\right\|+\left(1-\gamma_{n}\right)\left\|y_{n}-x^{*}\right\| \\
& \leq\left\|x_{n}-x^{*}\right\| .
\end{aligned}
$$


International Journal of Mathematics and Mathematical Sciences

From (2.16), by the contraction of $f$ and the nonexpansivity of $W_{n}$, we have

$$
\begin{aligned}
\left\|x_{n+1}-x^{*}\right\|= & \left\|\alpha_{n} \gamma f\left(W_{n} x_{n}\right)+\beta_{n} x_{n}+\left(\left(1-\beta_{n}\right) I-\alpha_{n} A\right) t_{n}-x^{*}\right\| \\
= & \left\|\alpha_{n}\left(\gamma f\left(W_{n} x_{n}\right)-A x^{*}\right)+\beta_{n}\left(x_{n}-x^{*}\right)+\left(\left(1-\beta_{n}\right) I-\alpha_{n} A\right)\left(t_{n}-x^{*}\right)\right\| \\
\leq & \alpha_{n}\left\|\gamma f\left(W_{n} x_{n}\right)-A x^{*}\right\|+\beta_{n}\left\|x_{n}-x^{*}\right\|+\left\|\left(1-\beta_{n}\right) I-\alpha_{n} A\right\|\left\|t_{n}-x^{*}\right\| \\
\leq & \alpha_{n} \gamma\left\|f\left(W_{n} x_{n}\right)-f\left(x^{*}\right)\right\|+\alpha_{n}\left\|\gamma f\left(x^{*}\right)-A x^{*}\right\|+\beta_{n}\left\|x_{n}-x^{*}\right\| \\
& +\left(1-\beta_{n}-\alpha_{n} \bar{\delta}\right)\left\|x_{n}-x^{*}\right\| \\
\leq & \alpha_{n} \gamma \delta\left\|W_{n} x_{n}-x^{*}\right\|+\alpha_{n}\left\|\gamma f\left(x^{*}\right)-A x^{*}\right\|+\left(1-\alpha_{n} \bar{\delta}\right)\left\|x_{n}-x^{*}\right\| \\
\leq & \left(1-(\bar{\delta}-\gamma \delta) \alpha_{n}\right)\left\|x_{n}-x^{*}\right\|+\alpha_{n}\left\|\gamma f\left(x^{*}\right)-A x^{*}\right\| \\
\leq & \max \left\{\left\|x_{n}-x^{*}\right\|, \frac{\left\|\gamma f\left(x^{*}\right)-A x^{*}\right\|}{\bar{\delta}-\gamma \delta}\right\} .
\end{aligned}
$$

It follows from induction that

$$
\left\|x_{n+1}-x^{*}\right\| \leq \max \left\{\left\|x_{1}-x^{*}\right\|, \frac{\left\|\gamma f\left(x^{*}\right)-A x^{*}\right\|}{\bar{\delta}-\gamma \delta}\right\}
$$

for all $n \in \mathbb{N}$. Hence, $\left\{x_{n}\right\}$ is bounded, and so are $\left\{y_{n}\right\},\left\{z_{n}\right\},\left\{t_{n}\right\},\left\{W_{n} x_{n}\right\},\left\{f\left(W_{n} x_{n}\right)\right\}$ and $\left\{A t_{n}\right\}$.

Next, we prove that $\left\|v_{n}-x_{n}\right\| \rightarrow 0,\left\|x_{n+1}-x_{n}\right\| \rightarrow 0$ and $\left\|t_{n}-x_{n}\right\| \rightarrow 0$ as $n \rightarrow \infty$. By the nonexpansivity of $J_{M_{i}, \lambda_{i}}$ and $I-\lambda_{i}\left(B_{i}+C_{i}\right)$, we have

$$
\begin{aligned}
\left\|y_{n+1}-y_{n}\right\| & =\left\|J_{M_{1}, \lambda_{1}}\left(z_{n+1}-\lambda_{1}\left(B_{1}+C_{1}\right) z_{n+1}\right)-J_{M_{1}, \lambda_{1}}\left(z_{n}-\lambda_{1}\left(B_{1}+C_{1}\right) z_{n}\right)\right\| \\
& \leq\left\|\left(z_{n+1}-\lambda_{1}\left(B_{1}+C_{1}\right) z_{n+1}\right)-\left(z_{n}-\lambda_{1}\left(B_{1}+C_{1}\right) z_{n}\right)\right\| \\
& \leq\left\|z_{n+1}-z_{n}\right\| .
\end{aligned}
$$

Similarly, we have

$$
\left\|z_{n+1}-z_{n}\right\| \leq\left\|x_{n+1}-x_{n}\right\|
$$

Therefore, from (2.19) and (2.20), we have

$$
\left\|y_{n+1}-y_{n}\right\| \leq\left\|z_{n+1}-z_{n}\right\| \leq\left\|x_{n+1}-x_{n}\right\|
$$


By the nonexpansivity of $S_{i}$ and $U_{n, i}$, we have

$$
\begin{aligned}
\left\|W_{n+1} x_{n}-W_{n} x_{n}\right\| & =\left\|U_{n+1,1} x_{n}-U_{n, 1} x_{n}\right\| \\
& =\left\|\mu_{1} S_{1} U_{n+1,2} x_{n}+\left(1-\mu_{1}\right) x_{n}-\left(\mu_{1} S_{1} U_{n, 2} x_{n}+\left(1-\mu_{1}\right) x_{n}\right)\right\| \\
& \leq \mu_{1}\left\|U_{n+1,2} x_{n}-U_{n, 2} x_{n}\right\| \\
& =\mu_{1}\left\|\mu_{2} S_{2} U_{n+1,3} x_{n}+\left(1-\mu_{2}\right) x_{n}-\left(\mu_{2} S_{2} U_{n, 3} x_{n}+\left(1-\mu_{2}\right) x_{n}\right)\right\| \\
& \leq \mu_{1} \mu_{2}\left\|U_{n+1,3} x_{n}-U_{n, 3} x_{n}\right\| \\
& \vdots \\
& \leq\left(\prod_{i=1}^{n} \mu_{i}\right)\left\|U_{n+1, n+1} x_{n}-U_{n, n+1} x_{n}\right\| \\
& \leq M \prod_{i=1}^{n} \mu_{i}
\end{aligned}
$$

for some constant $M$ such that $M \geq\left\|U_{n+1, n+1} x_{n}-U_{n, n+1} x_{n}\right\| \geq 0$. Therefore, from (2.22), by the nonexpansivity of $W_{n+1}$, we have

$$
\begin{aligned}
\left\|W_{n+1} x_{n+1}-W_{n} x_{n}\right\| & \leq\left\|W_{n+1} x_{n+1}-W_{n+1} x_{n}\right\|+\left\|W_{n+1} x_{n}-W_{n} x_{n}\right\| \\
& \leq\left\|x_{n+1}-x_{n}\right\|+M \prod_{i=1}^{n} \mu_{i} .
\end{aligned}
$$

Since

$$
\begin{aligned}
t_{n+1}-t_{n}= & \gamma_{n+1} W_{n+1} x_{n+1}+\left(1-\gamma_{n+1}\right) y_{n+1}-\left(\gamma_{n} W_{n} x_{n}+\left(1-\gamma_{n}\right) y_{n}\right) \\
= & \gamma_{n+1}\left(W_{n+1} x_{n+1}-W_{n} x_{n}\right)+\left(\gamma_{n+1}-\gamma_{n}\right)\left(W_{n} x_{n}-y_{n}\right) \\
& +\left(1-\gamma_{n+1}\right)\left(y_{n+1}-y_{n}\right),
\end{aligned}
$$

combining (2.21), (2.23), and (2.24), we have

$$
\begin{aligned}
\left\|t_{n+1}-t_{n}\right\| & \leq \gamma_{n+1}\left\|W_{n+1} x_{n+1}-W_{n} x_{n}\right\|+\left|\gamma_{n+1}-\gamma_{n}\right|\left\|W_{n} x_{n}-y_{n}\right\|+\left(1-\gamma_{n+1}\right)\left\|y_{n+1}-y_{n}\right\| \\
& \leq \gamma_{n+1}\left(\left\|x_{n+1}-x_{n}\right\|+M \prod_{i=1}^{n} \mu_{i}\right)+\left|\gamma_{n+1}-\gamma_{n}\right|\left\|W_{n} x_{n}-y_{n}\right\|+\left(1-\gamma_{n+1}\right)\left\|y_{n+1}-y_{n}\right\| \\
& \leq M \prod_{i=1}^{n} \mu_{i}+\left|\gamma_{n+1}-\gamma_{n}\right|\left\|W_{n} x_{n}-y_{n}\right\|+\left\|x_{n+1}-x_{n}\right\| .
\end{aligned}
$$


International Journal of Mathematics and Mathematical Sciences

Let $v_{n}=\left(x_{n+1}-\beta_{n} x_{n}\right) /\left(1-\beta_{n}\right)$. Then we have

$$
x_{n+1}=\left(1-\beta_{n}\right) v_{n}+\beta_{n} x_{n} .
$$

Since

$$
\begin{aligned}
v_{n+1}-v_{n}= & \frac{\alpha_{n+1} \gamma f\left(W_{n+1} x_{n+1}\right)+\left(\left(1-\beta_{n+1}\right) I-\alpha_{n+1} A\right) t_{n+1}}{1-\beta_{n+1}} \\
& -\frac{\alpha_{n} \gamma f\left(W_{n} x_{n}\right)+\left(\left(1-\beta_{n}\right) I-\alpha_{n} A\right) t_{n}}{1-\beta_{n}} \\
= & \frac{\alpha_{n+1}}{1-\beta_{n+1}}\left(\gamma f\left(W_{n+1} x_{n+1}\right)-A t_{n+1}\right)-\frac{\alpha_{n}}{1-\beta_{n}}\left(\gamma f\left(W_{n} x_{n}\right)-A t_{n}\right)+\left(t_{n+1}-t_{n}\right),
\end{aligned}
$$

combining (2.25) and (2.27), we have

$$
\begin{aligned}
\left\|v_{n+1}-v_{n}\right\| \leq & \frac{\alpha_{n+1}}{1-\beta_{n+1}}\left\|\gamma f\left(W_{n+1} x_{n+1}\right)-A t_{n+1}\right\|+\frac{\alpha_{n}}{1-\beta_{n}}\left\|\gamma f\left(W_{n} x_{n}\right)-A t_{n}\right\|+\left\|t_{n+1}-t_{n}\right\| \\
\leq & \frac{\alpha_{n+1}}{1-\beta_{n+1}}\left\|\gamma f\left(W_{n+1} x_{n+1}\right)-A t_{n+1}\right\|+\frac{\alpha_{n}}{1-\beta_{n}}\left\|\gamma f\left(W_{n} x_{n}\right)-A t_{n}\right\| \\
& +M \prod_{i=1}^{n} \mu_{i}+\left|\gamma_{n+1}-\gamma_{n}\right|\left\|W_{n} x_{n}-y_{n}\right\|+\left\|x_{n+1}-x_{n}\right\| .
\end{aligned}
$$

Therefore, by (C1), (C2) and $\lim _{n \rightarrow \infty} \prod_{i=1}^{n} \mu_{i}=0$, we get

$$
\limsup _{n \rightarrow \infty}\left(\left\|v_{n+1}-v_{n}\right\|-\left\|x_{n+1}-x_{n}\right\|\right) \leq 0 .
$$

From (2.26) and (2.29), by (C2) and Lemma 1.3, we obtain

$$
\left\|v_{n}-x_{n}\right\| \longrightarrow 0 \text { as } n \longrightarrow \infty
$$

From (2.26), by (2.30), we obtain

$$
\left\|x_{n+1}-x_{n}\right\|=\left(1-\beta_{n}\right)\left\|v_{n}-x_{n}\right\| \longrightarrow 0 \text { as } n \longrightarrow \infty .
$$

Since

$$
\begin{aligned}
x_{n+1}-x_{n} & =\alpha_{n} \gamma f\left(W_{n} x_{n}\right)+\beta_{n} x_{n}+\left(\left(1-\beta_{n}\right) I-\alpha_{n} A\right) t_{n}-x_{n} \\
& =\alpha_{n}\left(\gamma f\left(W_{n} x_{n}\right)-A t_{n}\right)+\left(1-\beta_{n}\right)\left(t_{n}-x_{n}\right),
\end{aligned}
$$


therefore,

$$
\left(1-\beta_{n}\right)\left\|t_{n}-x_{n}\right\| \leq \alpha_{n}\left\|\gamma f\left(W_{n} x_{n}\right)-A t_{n}\right\|+\left\|x_{n+1}-x_{n}\right\| .
$$

From (2.31), by (C1) and (C2), we obtain

$$
\left\|t_{n}-x_{n}\right\| \longrightarrow 0 \quad \text { as } n \longrightarrow \infty \text {. }
$$

For all $x, y \in H$, by Lemma 1.2(2), the nonexpansivity of $P_{\Omega}$ and the contraction of $f$, we have

$$
\begin{aligned}
\left\|P_{\Omega}(I-A+\gamma f) x-P_{\Omega}(I-A+\gamma f) y\right\| & \leq\|(I-A+\gamma f) x-(I-A+\gamma f) y\| \\
& \leq \gamma\|f(\mathrm{x})-f(y)\|+\|I-A\|\|x-y\| \\
& \leq \gamma \delta\|x-y\|+(1-\bar{\delta})\|x-y\| \\
& =(1-(\bar{\delta}-\gamma \delta))\|x-y\| .
\end{aligned}
$$

Therefore, $P_{\Omega}(I-A+\gamma f)$ is a contraction with coefficient $1-(\bar{\delta}-\gamma \delta)$, by Banach contraction principle guarantees that $P_{\Omega}(I-A+\gamma f)$ has a unique fixed point, say $w \in H$, that is, $w=$ $P_{\Omega}(I-A+\gamma f) w$.

Next, we claim that

$$
\limsup _{n \rightarrow \infty}\left\langle\gamma f(w)-A w, x_{n}-w\right\rangle \leq 0
$$

To show this inequality, we choose a subsequence $\left\{x_{n_{i}}\right\}$ of $\left\{x_{n}\right\}$ such that

$$
\limsup _{n \rightarrow \infty}\left\langle\gamma f(w)-A w, x_{n}-w\right\rangle=\lim _{i \rightarrow \infty}\left\langle\gamma f(w)-A w, x_{n_{i}}-w\right\rangle
$$

Since, $\left\{x_{n_{i}}\right\}$ is bounded, there exists a subsequence $\left\{x_{n_{i}}\right\}$ of $\left\{x_{n_{i}}\right\}$ which converges weakly to $\bar{w}$. Without loss of generality, we can assume that $x_{n_{i}} \rightarrow \bar{w}$ as $i \rightarrow \infty$.

Next, we prove that $\bar{w} \in \Omega$. Define sequence of mappings $\left\{R_{n}: H \rightarrow \mathrm{H}\right\}$ and mapping $R: H \rightarrow H$ by

$$
\begin{gathered}
R_{n} x=\gamma_{n} W_{n} x+\left(1-\gamma_{n}\right) D x, \quad \forall n \in \mathbb{N}, \\
R x=\lim _{n \rightarrow \infty} R_{n} x .
\end{gathered}
$$

Thus, by Lemma 1.5(3) and (C3), we have

$$
R x=a W x+(1-a) D x
$$


where $0<a=\lim _{n \rightarrow \infty} \gamma_{n}<1$. Since, $W$ and $D$ are nonexpansive and by Lemma 1.5(3), $F(W)=\bigcap_{i=1}^{\infty} F\left(S_{i}\right)$. Therefore, by Lemma 1.9, we get $R$ is a nonexpansive and $F(R)=F(W) \cap$ $F(D)=\bigcap_{i=1}^{\infty} F\left(S_{i}\right) \cap F(D)$. Since, $S_{n} x=\delta_{n} x+\left(1-\delta_{n}\right) T_{n} x$, where $\delta_{n} \in\left[k_{n}, 1\right)$. Thus, by Lemma 1.11, we obtain

$$
F(R)=\bigcap_{i=1}^{\infty} F\left(S_{i}\right) \cap F(D)=\bigcap_{i=1}^{\infty} F\left(T_{i}\right) \cap F(D)=\Omega .
$$

From (2.34), we have $\left\|t_{n_{i}}-x_{n_{i}}\right\| \rightarrow 0$ as $i \rightarrow \infty$. Thus, from (2.38), we get $\left\|R x_{n_{i}}-x_{n_{i}}\right\| \rightarrow 0$ as $i \rightarrow \infty$. It follows from $x_{n_{i}}-\bar{w}$ and by Lemma 1.10 that $\bar{w} \in F(R)$, that is $\bar{w} \in \Omega$. Therefore, from (2.37), we obtain

$$
\begin{aligned}
\limsup _{n \rightarrow \infty}\left\langle\gamma f(w)-A w, x_{n}-w\right\rangle & =\lim _{i \rightarrow \infty}\left\langle\gamma f(w)-A w, x_{n_{i}}-w\right\rangle \\
& =\langle(\gamma f-A) w, \bar{w}-w\rangle \leq 0 .
\end{aligned}
$$

Next, we prove that $x_{n} \rightarrow w$ as $n \rightarrow \infty$. Since $w \in \Omega$, we have $W_{n} w=w$, and the same as in (2.16), we have

$$
\left\|t_{n}-w\right\| \leq\left\|x_{n}-w\right\|
$$

It follows by the contraction of $f$ and the nonexpansivity of $W_{n}$ that

$$
\begin{aligned}
\left\|x_{n+1}-w\right\|^{2}= & \left\|\alpha_{n} \gamma f\left(W_{n} x_{n}\right)+\beta_{n} x_{n}+\left(\left(1-\beta_{n}\right) I-\alpha_{n} A\right) t_{n}-w\right\|^{2} \\
= & \left\|\left(\left(1-\beta_{n}\right) I-\alpha_{n} A\right)\left(t_{n}-w\right)+\beta_{n}\left(x_{n}-w\right)+\alpha_{n}\left(\gamma f\left(W_{n} x_{n}\right)-A w\right)\right\| \\
= & \left\|\left(\left(1-\beta_{n}\right) I-\alpha_{n} A\right)\left(t_{n}-w\right)+\beta_{n}\left(x_{n}-w\right)\right\|^{2}+\alpha_{n}^{2}\left\|\gamma f\left(W_{n} x_{n}\right)-A w\right\|^{2} \\
& +2 \alpha_{n} \beta_{n}\left\langle x_{n}-w, \gamma f\left(W_{n} x_{n}\right)-A w\right\rangle \\
& +2 \alpha_{n}\left\langle\left(\left(1-\beta_{n}\right) I-\alpha_{n} A\right)\left(t_{n}-w\right), \gamma f\left(W_{n} x_{n}\right)-A w\right\rangle \\
\leq & \left(\left(1-\beta_{n}-\alpha_{n} \bar{\delta}\right)\left\|t_{n}-w\right\|+\beta_{n}\left\|x_{n}-w\right\|\right)^{2}+\alpha_{n}^{2}\left\|\gamma f\left(W_{n} x_{n}\right)-A w\right\|^{2} \\
& +2 \alpha_{n} \beta_{n} \gamma\left\langle x_{n}-w, f\left(W_{n} x_{n}\right)-f(w)\right\rangle+2 \alpha_{n} \beta_{n}\left\langle x_{n}-w, \gamma f(w)-A w\right\rangle \\
& +2 \alpha_{n}\left(1-\beta_{n}\right) \gamma\left\langle t_{n}-w, f\left(W_{n} x_{n}\right)-f(w)\right\rangle \\
& +2 \alpha_{n}\left(1-\beta_{n}\right)\left\langle t_{n}-w, \gamma f(w)-A w\right\rangle \\
& -2 \alpha_{n}^{2}\left\langle A\left(t_{n}-w\right), \gamma f\left(W_{n} x_{n}\right)-A w\right\rangle
\end{aligned}
$$




$$
\begin{aligned}
\leq & \left(1-\alpha_{n} \bar{\delta}\right)^{2}\left\|x_{n}-w\right\|^{2}+2 \alpha_{n} \beta_{n} \gamma\left\|x_{n}-w\right\|\left\|f\left(W_{n} x_{n}\right)-f(w)\right\| \\
& +2 \alpha_{n}\left(1-\beta_{n}\right) \gamma\left\|t_{n}-w\right\|\left\|f\left(W_{n} x_{n}\right)-f(w)\right\|+\theta_{n} \\
\leq & \left(1-\alpha_{n} \bar{\delta}\right)^{2}\left\|x_{n}-w\right\|^{2}+2 \alpha_{n} \beta_{n} \gamma \delta\left\|x_{n}-w\right\|^{2}+2 \alpha_{n}\left(1-\beta_{n}\right) \gamma \delta\left\|x_{n}-w\right\|^{2}+\theta_{n} \\
= & \left(1-\alpha_{n} \bar{\delta}\right)^{2}\left\|x_{n}-w\right\|^{2}+2 \alpha_{n} \gamma \delta\left\|x_{n}-w\right\|^{2}+\theta_{n} \\
= & \left(1-2(\bar{\delta}-\gamma \delta) \alpha_{n}\right)\left\|x_{n}-w\right\|^{2}+\alpha_{n}^{2} \bar{\delta}^{2}\left\|x_{n}-w\right\|^{2}+\theta_{n} \\
\leq & \left(1-\eta_{n}\right)\left\|x_{n}-w\right\|^{2}+\delta_{n}^{\prime}
\end{aligned}
$$

where $\eta_{n}:=(\bar{\delta}-\gamma \delta) \alpha_{n} \in(0,1), \delta_{n}^{\prime}:=\alpha_{n}^{2} \bar{\delta}^{2}\left\|x_{n}-w\right\|^{2}+\theta_{n}$ and

$$
\begin{aligned}
\theta_{n}:= & \alpha_{n}^{2}\left\|\gamma f\left(W_{n} x_{n}\right)-A w\right\|^{2}+2 \alpha_{n} \beta_{n}\left\langle x_{n}-w, \gamma f(w)-A w\right\rangle \\
& +2 \alpha_{n}\left(1-\beta_{n}\right)\left\langle t_{n}-w, \gamma f(w)-A w\right\rangle-2 \alpha_{n}^{2}\left\langle A\left(t_{n}-w\right), \gamma f\left(W_{n} x_{n}\right)-A w\right\rangle .
\end{aligned}
$$

By (2.34), (2.41), (C1) and (C3), we can found that $\sum_{n=1}^{\infty} \eta_{n}=\infty$ and $\lim \sup _{n \rightarrow \infty}\left(\delta_{n}^{\prime} / \eta_{n}\right) \leq 0$. By Lemma 1.4, we obtain $\left\{x_{n}\right\}$ converges strongly to $w$. This proof is completed.

Remarks 2.2. Theorem 2.1 improve and extend to the main results of $\mathrm{Li}$ and $\mathrm{Wu}$ [12] for solving the generalized system of relaxed cocoercive quasivariational inclusions and fixed points problems of an infinite family of strictly pseudocontractive mappings.

\section{Applications}

Theorem 3.1. Let $H$ be a real Hilbert space, $M: H \rightarrow 2^{H}$ be a maximal monotone mapping, $B: H \rightarrow H$ be a relaxed $(\alpha, r)$-cocoercive and $\xi$-Lipschitz continuous mappings, respectively, $C$ : $H \rightarrow H$ be a relaxed $(\bar{\alpha}, \bar{r})$-cocoercive and $\bar{\xi}$-Lipschitz continuous mappings, respectively. Let $A$ : $H \rightarrow H$ be a strongly positive linear bounded self-adjoint operator mapping with coefficient $\bar{\delta} \in(0,1]$ such that $\|A\| \leq 1$ and $f: H \rightarrow H$ be a contraction mapping with coefficient $\delta \in(0,1)$. Let $\left\{T_{n}: H \rightarrow H\right\}$ be a family of $k_{n}$-strictly pseudocontractive mappings with a fixed point such that $k_{n} \in[0,1)$ for all $n \in \mathbb{N}$. Define $S_{n} x=\delta_{n} x+\left(1-\delta_{n}\right) T_{n} x$, where $\delta_{n} \in\left[k_{n}, 1\right)$, for all $n \in \mathbb{N}$, and let $W_{n}: H \rightarrow H$ be a $W$-mapping generated by $\left\{S_{n}\right\}$ and $\left\{\mu_{n}\right\}$ such that $\left\{\mu_{n}\right\} \subset(0, \mu]$, for some $\mu \in(0,1)$. Assume that $\Omega:=\bigcap_{n=1}^{\infty} F\left(T_{n}\right) \cap F(D) \neq \emptyset$ and $0<\gamma<\bar{\delta} / \delta$ where $D: H \rightarrow H$ defined by $D x=J_{M, \lambda_{1}}\left(\left(I-\lambda_{1}(B+C)\right) J_{M, \lambda_{2}}\left(I-\lambda_{2}(B+C)\right) x\right)$. For $x_{1} \in H$, suppose that $\left\{x_{n}\right\}$ be generated iteratively by

$$
\begin{gathered}
z_{n}=J_{M, \lambda_{2}}\left(x_{n}-\lambda_{2}(B+C) x_{n}\right), \\
y_{n}=J_{M, \lambda_{1}}\left(z_{n}-\lambda_{1}(B+C) z_{n}\right), \\
x_{n+1}=\alpha_{n} \gamma f\left(W_{n} x_{n}\right)+\beta_{n} x_{n}+\left(\left(1-\beta_{n}\right) I-\alpha_{n} A\right)\left(\gamma_{n} W_{n} x_{n}+\left(1-\gamma_{n}\right) y_{n}\right),
\end{gathered}
$$


for all $n \in \mathbb{N}$, where $\left\{\alpha_{n}\right\},\left\{\beta_{n}\right\},\left\{\gamma_{n}\right\} \subset(0,1), \lambda_{i} \in(0, \epsilon], \epsilon=\min \left\{2\left(r-\alpha \xi^{2}\right) / \xi(\xi+\bar{\xi}), 2(\bar{r}-\right.$ $\left.\left.\bar{\alpha} \bar{\xi}^{2}\right) / \bar{\xi}(\xi+\bar{\xi})\right\}, r>\alpha \xi^{2}, \bar{r}>\bar{\alpha} \bar{\xi}^{2}$, for each $i=1,2$, satisfying the following conditions:

(C1) $\lim _{n \rightarrow \infty} \alpha_{n}=\lim _{n \rightarrow \infty}\left|\gamma_{n+1}-\gamma_{n}\right|=0$;

(C2) $0<\liminf _{n \rightarrow \infty} \beta_{n} \leq \lim \sup _{n \rightarrow \infty} \beta_{n}<1$;

(C3) $\sum_{n=1}^{\infty} \alpha_{n}=\infty$ and $0<\lim _{n \rightarrow \infty} \gamma_{n}<1$.

Then the sequence $\left\{x_{n}\right\}$ converges strongly to $w \in \Omega$ where $w=P_{\Omega}(I-A+\gamma f)$ w is a unique solution of the variational inequality

$$
\langle(A-\gamma f) w, y-w\rangle \geq 0, \quad \forall y \in \Omega
$$

which is the optimality condition for the minimization problem

$$
\min _{x \in \Omega}\left\{\frac{1}{2}\langle A x, x\rangle-h(x)\right\}
$$

where $h$ is a potential function for $\gamma f$ (i.e., $h^{\prime}(x)=\gamma f(x)$ for $\left.x \in H\right)$ and $\left(w, J_{M, \lambda_{2}}\left(I-\lambda_{2}(B+C)\right) w\right.$ ) is a solution of problem (1.19).

Proof. It is concluded obviously, from Theorem 2.1 by putting $M_{1}=M_{2}=M, B_{1}=B_{2}=B$ and $C_{1}=C_{2}=C$.

Theorem 3.2. Let $H$ be a real Hilbert space, $M: H \rightarrow 2^{H}$ be a maximal monotone mapping, $B$ : $H \rightarrow H$ be a relaxed $(\alpha, r)$-cocoercive and $\xi$-Lipschitz continuous mappings, respectively, $C: H \rightarrow$ $H$ be a relaxed $(\bar{\alpha}, \bar{r})$-cocoercive and $\bar{\xi}$-Lipschitz continuous mappings, respectively. Let $A: H \rightarrow H$ be a strongly positive linear bounded self-adjoint operator mapping with coefficient $\bar{\delta} \in(0,1]$ such that $\|A\| \leq 1$ and $f: H \rightarrow H$ be a contraction mapping with coefficient $\delta \in(0,1)$. Let $\left\{T_{n}: H \rightarrow H\right\}$ be a family of nonexpansive mappings. Define $S_{n} x=\delta_{n} x+\left(1-\delta_{n}\right) T_{n} x$, where $\delta_{n} \in[0,1)$, for all $n \in \mathbb{N}$, and let $W_{n}: H \rightarrow H$ be a $W$-mapping generated by $\left\{S_{n}\right\}$ and $\left\{\mu_{n}\right\}$ such that $\left\{\mu_{n}\right\} \subset(0, \mu]$, for some $\mu \in(0,1)$. Assume that $\Omega:=\bigcap_{n=1}^{\infty} F\left(T_{n}\right) \cap F(D) \neq \emptyset$ and $0<\gamma<\bar{\delta} / \delta$ where $D: H \rightarrow H$ defined by $D x=J_{M, \lambda_{1}}\left(\left(I-\lambda_{1}(B+C)\right) J_{M, \lambda_{2}}\left(I-\lambda_{2}(B+C)\right) x\right)$. For $x_{1} \in H$, suppose that $\left\{x_{n}\right\}$ be generated iteratively by

$$
\begin{gathered}
z_{n}=J_{M, \lambda_{2}}\left(x_{n}-\lambda_{2}(B+C) x_{n}\right), \\
y_{n}=J_{M, \lambda_{1}}\left(z_{n}-\lambda_{1}(B+C) z_{n}\right), \\
x_{n+1}=\alpha_{n} \gamma f\left(W_{n} x_{n}\right)+\beta_{n} x_{n}+\left(\left(1-\beta_{n}\right) I-\alpha_{n} A\right)\left(\gamma_{n} W_{n} x_{n}+\left(1-\gamma_{n}\right) y_{n}\right),
\end{gathered}
$$

for all $n \in \mathbb{N}$, where $\left\{\alpha_{n}\right\},\left\{\beta_{n}\right\},\left\{\gamma_{n}\right\} \subset(0,1), \lambda_{i} \in(0, \epsilon], \epsilon=\min \left\{2\left(r-\alpha \xi^{2}\right) / \xi(\xi+\bar{\xi}), 2(\bar{r}-\right.$ $\left.\left.\bar{\alpha} \bar{\xi}^{2}\right) / \bar{\xi}(\xi+\bar{\xi})\right\}, r>\alpha \xi^{2}, \bar{r}>\bar{\alpha} \bar{\xi}^{2}$, for each $i=1,2$, satisfying the following conditions:

(C1) $\lim _{n \rightarrow \infty} \alpha_{n}=\lim _{n \rightarrow \infty}\left|\gamma_{n+1}-\gamma_{n}\right|=0$;

(C2) $0<\liminf _{n \rightarrow \infty} \beta_{n} \leq \lim \sup _{n \rightarrow \infty} \beta_{n}<1$;

(C3) $\sum_{n=1}^{\infty} \alpha_{n}=\infty$ and $0<\lim _{n \rightarrow \infty} \gamma_{n}<1$. 
Then the sequence $\left\{x_{n}\right\}$ converges strongly to $w \in \Omega$ where $w=P_{\Omega}(I-A+\gamma f)$ w is a unique solution of the variational inequality

$$
\langle(A-\gamma f) w, y-w\rangle \geq 0, \quad \forall y \in \Omega,
$$

which is the optimality condition for the minimization problem

$$
\min _{x \in \Omega}\left\{\frac{1}{2}\langle A x, x\rangle-h(x)\right\}
$$

where $h$ is a potential function for $\gamma f$ (i.e., $h^{\prime}(x)=\gamma f(x)$ for $\left.x \in H\right)$ and $\left(w, J_{M, \lambda_{2}}\left(I-\lambda_{2}(B+C)\right) w\right.$ ) is a solution of problem (1.19).

Proof. It is concluded obviously, from Theorem 3.1 by putting $k_{n}=0$ for all $n \in \mathbb{N}$.

Theorem 3.3. Let $H$ be a real Hilbert space, $M_{i}: H \rightarrow 2^{H}$ be a maximal monotone mapping, $B_{i}: H \rightarrow H$ be a relaxed $\left(\alpha_{i}, r_{i}\right)$-cocoercive and $\xi_{i}$-Lipschitz continuous mappings, respectively, for each $i=1,2$. Let $A: H \rightarrow H$ be a strongly positive linear bounded self-adjoint operator mapping with coefficient $\bar{\delta} \in(0,1]$ such that $\|A\| \leq 1$ and $f: H \rightarrow H$ be a contraction mapping with coefficient $\delta \in(0,1)$. Let $\left\{T_{n}: H \rightarrow H\right\}$ be a family of $k_{n}$-strictly pseudocontractive mappings with a fixed point such that $k_{n} \in[0,1)$ for all $n \in \mathbb{N}$. Define $S_{n} x=\delta_{n} x+\left(1-\delta_{n}\right) T_{n} x$, where $\delta_{n} \in\left[k_{n}, 1\right)$, for all $n \in \mathbb{N}$, and let $W_{n}: H \rightarrow H$ be a $W$-mapping generated by $\left\{S_{n}\right\}$ and $\left\{\mu_{n}\right\}$ such that $\left\{\mu_{n}\right\} \subset(0, \mu]$, for some $\mu \in(0,1)$. Assume that $\Omega:=\bigcap_{n=1}^{\infty} F\left(T_{n}\right) \cap F(D) \neq \emptyset$ and $0<\gamma<\bar{\delta} / \delta$ where $D: H \rightarrow H$ defined by $D x=J_{M_{1}, \lambda_{1}}\left(\left(I-\rho_{1} B_{1}\right) J_{M_{2}, \lambda_{2}}\left(I-\rho_{2} B_{2}\right) x\right)$ such that $\lambda_{i}=\rho_{i} / 2$ for each $i=1$, 2. For $x_{1} \in H$, suppose that $\left\{x_{n}\right\}$ be generated iteratively by

$$
\begin{gathered}
z_{n}=J_{M_{2}, \lambda_{2}}\left(x_{n}-\rho_{2} B_{2} x_{n}\right), \\
y_{n}=J_{M_{1}, \lambda_{1}}\left(z_{n}-\rho_{1} B_{1} z_{n}\right), \\
x_{n+1}=\alpha_{n} \gamma f\left(W_{n} x_{n}\right)+\beta_{n} x_{n}+\left(\left(1-\beta_{n}\right) I-\alpha_{n} A\right)\left(\gamma_{n} W_{n} x_{n}+\left(1-\gamma_{n}\right) y_{n}\right),
\end{gathered}
$$

for all $n \in \mathbb{N}$, where $\left\{\alpha_{n}\right\},\left\{\beta_{n}\right\},\left\{\gamma_{n}\right\} \subset(0,1), \rho_{i} \in\left(0,2\left(r_{i}-\alpha_{i} \xi_{i}^{2}\right) / \xi_{i}^{2}\right], r_{i}>\alpha_{i} \xi_{i}^{2}$, for each $i=1,2$, satisfying the following conditions:

(C1) $\lim _{n \rightarrow \infty} \alpha_{n}=\lim _{n \rightarrow \infty}\left|\gamma_{n+1}-\gamma_{n}\right|=0$;

(C2) $0<\liminf _{n \rightarrow \infty} \beta_{n} \leq \lim \sup _{n \rightarrow \infty} \beta_{n}<1$;

(C3) $\sum_{n=1}^{\infty} \alpha_{n}=\infty$ and $0<\lim _{n \rightarrow \infty} \gamma_{n}<1$.

Then the sequence $\left\{x_{n}\right\}$ converges strongly to $w \in \Omega$ where $w=P_{\Omega}(I-A+\gamma f) w$ is a unique solution of the variational inequality

$$
\langle(A-\gamma f) w, y-w\rangle \geq 0, \quad \forall y \in \Omega
$$

which is the optimality condition for the minimization problem

$$
\min _{x \in \Omega}\left\{\frac{1}{2}\langle A x, x\rangle-h(x)\right\},
$$


where $h$ is a potential function for $\gamma f$ (i.e., $h^{\prime}(x)=\gamma f(x)$ for $x \in H$ ) and $\left(w, J_{M_{2}, \lambda_{2}}\left(I-\rho_{2} B_{2}\right) w\right.$ ) is a solution of problem

$$
\begin{aligned}
& 0 \in x^{*}-y^{*}+\lambda_{1}\left(2 B_{1} y^{*}+M_{1} x^{*}\right), \\
& 0 \in y^{*}-x^{*}+\lambda_{2}\left(2 B_{2} x^{*}+M_{2} y^{*}\right) .
\end{aligned}
$$

Proof. It is concluded obviously, from Theorem 2.1 by putting $B_{i}=C_{i}$ for each $i=1,2$.

Theorem 3.4. Let $H$ be a real Hilbert space, $M: H \rightarrow 2^{H}$ be a maximal monotone mapping, $B: H \rightarrow H$ be a relaxed $(\alpha, r)$-cocoercive and $\xi$-Lipschitz continuous mappings, respectively. Let $A: H \rightarrow H$ be a strongly positive linear bounded self-adjoint operator mapping with coefficient $\bar{\delta} \in(0,1]$ such that $\|A\| \leq 1$ and $f: H \rightarrow H$ be a contraction mapping with coefficient $\delta \in(0,1)$. Let $\left\{T_{n}: H \rightarrow H\right\}$ be a family of $k_{n}$-strictly pseudocontractive mappings with a fixed point such that $k_{n} \in[0,1)$ for all $n \in \mathbb{N}$. Define $S_{n} x=\delta_{n} x+\left(1-\delta_{n}\right) T_{n} x$, where $\delta_{n} \in\left[k_{n}, 1\right)$, for all $n \in \mathbb{N}$, and let $W_{n}: H \rightarrow H$ be a $W$-mapping generated by $\left\{S_{n}\right\}$ and $\left\{\mu_{n}\right\}$ such that $\left\{\mu_{n}\right\} \subset(0, \mu]$, for some $\mu \in(0,1)$. Assume that $\Omega:=\bigcap_{n=1}^{\infty} F\left(T_{n}\right) \cap F(D) \neq \emptyset$ and $0<\gamma<\bar{\delta} / \delta$ where $D: H \rightarrow H$ defined by $D x=J_{M, \lambda_{1}}\left(\left(I-\rho_{1} B\right) J_{M, \lambda_{2}}\left(I-\rho_{2} B\right) x\right)$ such that $\lambda_{i}=\rho_{i} / 2$ for each $i=1$, 2. For $x_{1} \in H$, suppose that $\left\{x_{n}\right\}$ be generated iteratively by

$$
\begin{gathered}
z_{n}=J_{M, \lambda_{2}}\left(x_{n}-\rho_{2} B x_{n}\right), \\
y_{n}=J_{M, \lambda_{1}}\left(z_{n}-\rho_{1} B z_{n}\right), \\
x_{n+1}=\alpha_{n} \gamma f\left(W_{n} x_{n}\right)+\beta_{n} x_{n}+\left(\left(1-\beta_{n}\right) I-\alpha_{n} A\right)\left(\gamma_{n} W_{n} x_{n}+\left(1-\gamma_{n}\right) y_{n}\right),
\end{gathered}
$$

for all $n \in \mathbb{N}$, where $\left\{\alpha_{n}\right\},\left\{\beta_{n}\right\},\left\{\gamma_{n}\right\} \subset(0,1), \rho_{i} \in\left(0,2\left(r-\alpha \xi^{2}\right) / \xi^{2}\right], r>\alpha \xi^{2}$, for each $i=1,2$, satisfying the following conditions:

(C1) $\lim _{n \rightarrow \infty} \alpha_{n}=\lim _{n \rightarrow \infty}\left|\gamma_{n+1}-\gamma_{n}\right|=0$;

(C2) $0<\liminf _{n \rightarrow \infty} \beta_{n} \leq \lim \sup _{n \rightarrow \infty} \beta_{n}<1$;

(C3) $\sum_{n=1}^{\infty} \alpha_{n}=\infty$ and $0<\lim _{n \rightarrow \infty} \gamma_{n}<1$.

Then the sequence $\left\{x_{n}\right\}$ converges strongly to $w \in \Omega$ where $w=P_{\Omega}(I-A+\gamma f) w$ is a unique solution of the variational inequality

$$
\langle(A-\gamma f) w, y-w\rangle \geq 0, \quad \forall y \in \Omega,
$$

which is the optimality condition for the minimization problem

$$
\min _{x \in \Omega}\left\{\frac{1}{2}\langle A x, x\rangle-h(x)\right\},
$$

where $h$ is a potential function for $\gamma f\left(\right.$ i.e., $h^{\prime}(x)=\gamma f(x)$ for $\left.x \in H\right)$ and $\left(w, J_{M, \lambda_{2}}\left(I-\rho_{2} B\right) w\right)$ is a solution of problem

$$
\begin{aligned}
& 0 \in x^{*}-y^{*}+\lambda_{1}\left(2 B y^{*}+M x^{*}\right), \\
& 0 \in y^{*}-x^{*}+\lambda_{2}\left(2 B x^{*}+M y^{*}\right) .
\end{aligned}
$$


Proof. It is concluded obviously, from Theorem 3.3 by putting $B_{1}=B_{2}=B$ and $M_{1}=M_{2}$ $=M$.

Theorem 3.5. Let $H$ be a real Hilbert space, $M: H \rightarrow 2^{H}$ be a maximal monotone mapping, $B: H \rightarrow H$ be a relaxed $(\alpha, r)$-cocoercive and $\xi$-Lipschitz continuous mappings, respectively. Let $A: H \rightarrow H$ be a strongly positive linear bounded self-adjoint operator mapping with coefficient $\bar{\delta} \in(0,1]$ such that $\|A\| \leq 1$ and $f: H \rightarrow H$ be a contraction mapping with coefficient $\delta \in(0,1)$. Let $\left\{T_{n}: H \rightarrow H\right\}$ be a family of nonexpansive mappings. Define $S_{n} x=\delta_{n} x+\left(1-\delta_{n}\right) T_{n} x$, where $\delta_{n} \in[0,1)$, for all $n \in \mathbb{N}$, and let $W_{n}: H \rightarrow H$ be a $W$-mapping generated by $\left\{S_{n}\right\}$ and $\left\{\mu_{n}\right\}$ such that $\left\{\mu_{n}\right\} \subset(0, \mu]$, for some $\mu \in(0,1)$. Assume that $\Omega:=\bigcap_{n=1}^{\infty} F\left(T_{n}\right) \cap F(D) \neq \emptyset$ and $0<\gamma<\bar{\delta} / \delta$ where $D: H \rightarrow H$ defined by $D x=J_{M, \lambda_{1}}\left(\left(I-\rho_{1} B\right) J_{M, \lambda_{2}}\left(I-\rho_{2} B\right) x\right)$ such that $\lambda_{i}=\rho_{i} / 2$ for each $i=1$, 2. For $x_{1} \in H$, suppose that $\left\{x_{n}\right\}$ be generated iteratively by

$$
\begin{gathered}
z_{n}=J_{M, \lambda_{2}}\left(x_{n}-\rho_{2} B x_{n}\right), \\
y_{n}=J_{M, \lambda_{1}}\left(z_{n}-\rho_{1} B z_{n}\right), \\
x_{n+1}=\alpha_{n} \gamma f\left(W_{n} x_{n}\right)+\beta_{n} x_{n}+\left(\left(1-\beta_{n}\right) I-\alpha_{n} A\right)\left(\gamma_{n} W_{n} x_{n}+\left(1-\gamma_{n}\right) y_{n}\right),
\end{gathered}
$$

for all $n \in \mathbb{N}$, where $\left\{\alpha_{n}\right\},\left\{\beta_{n}\right\},\left\{\gamma_{n}\right\} \subset(0,1), \rho_{i} \in\left(0,2\left(r-\alpha \xi^{2}\right) / \xi^{2}\right], r>\alpha \xi^{2}$, for each $i=1,2$, satisfying the following conditions:

$$
\begin{aligned}
& \text { (C1) } \lim _{n \rightarrow \infty} \alpha_{n}=\lim _{n \rightarrow \infty}\left|\gamma_{n+1}-\gamma_{n}\right|=0 ; \\
& \text { (C2) } 0<\liminf _{n \rightarrow \infty} \beta_{n} \leq \limsup _{n \rightarrow \infty} \beta_{n}<1 \text {; } \\
& \text { (C3) } \sum_{n=1}^{\infty} \alpha_{n}=\infty \text { and } 0<\lim _{n \rightarrow \infty} \gamma_{n}<1 .
\end{aligned}
$$

Then the sequence $\left\{x_{n}\right\}$ converges strongly to $w \in \Omega$ where $w=P_{\Omega}(I-A+\gamma f) w$ is a unique solution of the variational inequality

$$
\langle(A-\gamma f) w, y-w\rangle \geq 0, \quad \forall y \in \Omega
$$

which is the optimality condition for the minimization problem

$$
\min _{x \in \Omega}\left\{\frac{1}{2}\langle A x, x\rangle-h(x)\right\}
$$

where $h$ is a potential function for $\gamma f\left(i . e ., h^{\prime}(x)=\gamma f(x)\right.$ for $\left.x \in H\right)$ and $\left(w, J_{M, \lambda_{2}}\left(I-\rho_{2} B\right) w\right)$ is a solution of problem

$$
\begin{aligned}
& 0 \in x^{*}-y^{*}+\lambda_{1}\left(2 B y^{*}+M x^{*}\right), \\
& 0 \in y^{*}-x^{*}+\lambda_{2}\left(2 B x^{*}+M y^{*}\right) .
\end{aligned}
$$

Proof. It is concluded obviously, from Theorem 3.4 by putting $k_{n}=0$ for all $n \in \mathbb{N}$. 


\section{References}

[1] W. Takahashi, Nonlinear Functional Analysis: Fixed Point Theory and Its Application, Yokohama Publishers, Yokohama, Japan, 2000.

[2] K. Aoyama, Y. Kimura, W. Takahashi, and M. Toyoda, "Approximation of common fixed points of a countable family of nonexpansive mappings in a Banach space," Nonlinear Analysis: Theory, Methods E Applications, vol. 67, no. 8, pp. 2350-2360, 2007.

[3] H. H. Bauschke, "The approximation of fixed points of compositions of nonexpansive mappings in Hilbert space," Journal of Mathematical Analysis and Applications, vol. 202, no. 1, pp. 150-159, 1996.

[4] M. Shang, Y. Su, and X. Qin, "Strong convergence theorems for a finite family of nonexpansive mappings," Fixed Point Theory and Applications, vol. 2007, Article ID 76971, 9 pages, 2007.

[5] K. Shimoji and W. Takahashi, "Strong convergence to common fixed points of infinite nonexpansive mappings and applications," Taiwanese Journal of Mathematics, vol. 5, no. 2, pp. 387-404, 2001.

[6] G. Marino and H.-K. Xu, "A general iterative method for nonexpansive mappings in Hilbert spaces," Journal of Mathematical Analysis and Applications, vol. 318, no. 1, pp. 43-52, 2006.

[7] A. Moudafi, "Viscosity approximation methods for fixed-points problems," Journal of Mathematical Analysis and Applications, vol. 241, no. 1, pp. 46-55, 2000.

[8] M. A. Noor and K. I. Noor, "Sensitivity analysis for quasi-variational inclusions," Journal of Mathematical Analysis and Applications, vol. 236, no. 2, pp. 290-299, 1999.

[9] M. A. Noor, "Generalized set-valued variational inclusions and resolvent equations," Journal of Mathematical Analysis and Applications, vol. 228, no. 1, pp. 206-220, 1998.

[10] H. Iiduka and W. Takahashi, "Strong convergence theorems for nonexpansive mappings and inversestrongly monotone mappings," Nonlinear Analysis: Theory, Methods E Applications, vol. 61, no. 3, pp. 341-350, 2005.

[11] S.-S. Zhang, J. H. W. Lee, and C. K. Chan, "Algorithms of common solutions to quasi variational inclusion and fixed point problems," Applied Mathematics and Mechanics, vol. 29, no. 5, pp. 571-581, 2008.

[12] Y. Li and C. Wu, "On the convergence for an iterative method for quasivariational inclusions," Fixed Point Theory and Applications, vol. 2010, Article ID 278973, 11 pages, 2010.

[13] T. Suzuki, "A sufficient and necessary condition for halpern-type strong convergence to fixed points of nonexpansive mappings," Proceedings of the American Mathematical Society, vol. 135, no. 1, pp. 99106, 2007.

[14] H.-K. Xu, "Viscosity approximation methods for nonexpansive mappings," Journal of Mathematical Analysis and Applications, vol. 298, no. 1, pp. 279-291, 2004.

[15] S. Takahashi and W. Takahashi, "Viscosity approximation methods for equilibrium problems and fixed point problems in Hilbert spaces," Journal of Mathematical Analysis and Applications, vol. 331, no. 1, pp. 506-515, 2007.

[16] R. E. Bruck Jr., "Properties of fixed-point sets of nonexpansive mappings in Banach spaces," Transactions of the American Mathematical Society, vol. 179, pp. 251-262, 1973.

[17] F. E. Browder, "Convergence of approximants to fixed points of nonexpansive non-linear mappings in Banach spaces," Archive for Rational Mechanics and Analysis, vol. 24, pp. 82-90, 1967.

[18] F. E. Browder and W. V. Petryshyn, "Construction of fixed points of nonlinear mappings in Hilbert space," Journal of Mathematical Analysis and Applications, vol. 20, pp. 197-228, 1967. 


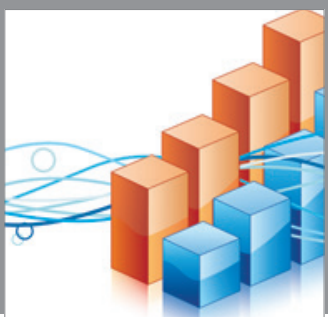

Advances in

Operations Research

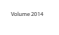

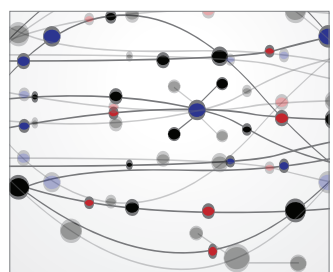

\section{The Scientific} World Journal
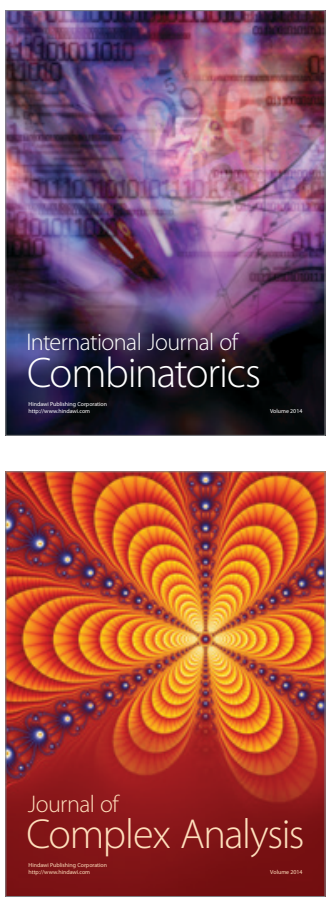

International Journal of

Mathematics and

Mathematical

Sciences
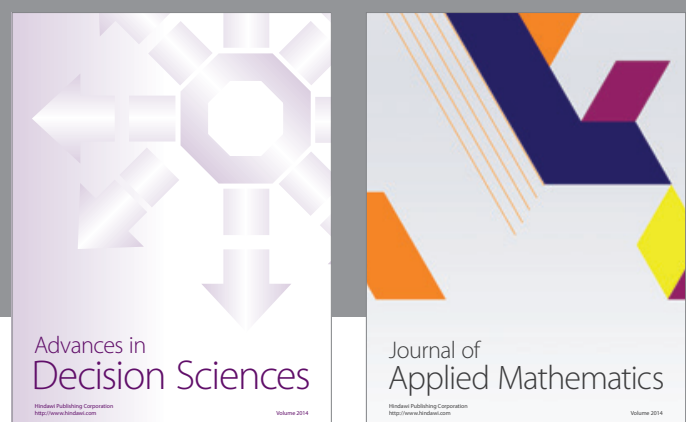

Journal of

Applied Mathematics
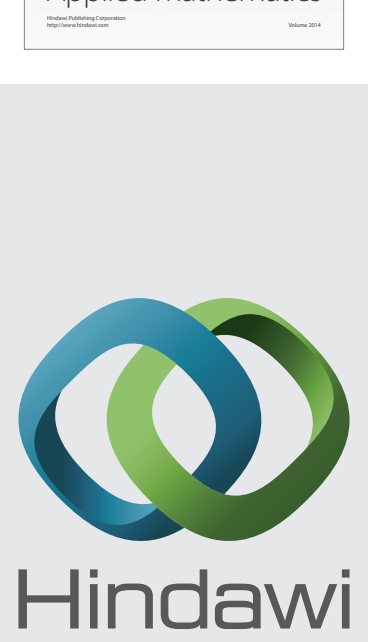

Submit your manuscripts at http://www.hindawi.com
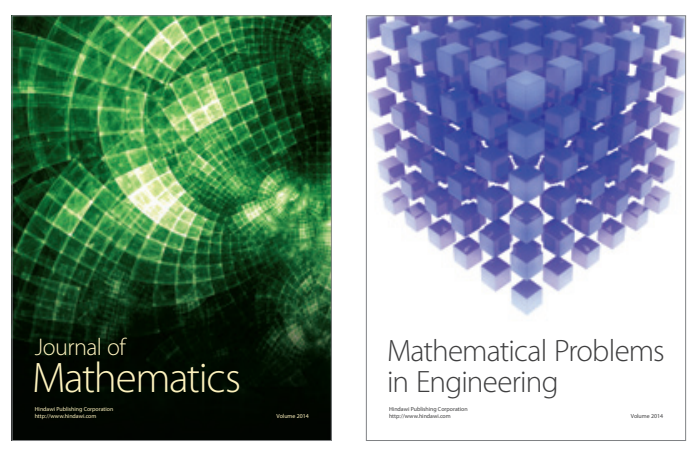

Mathematical Problems in Engineering
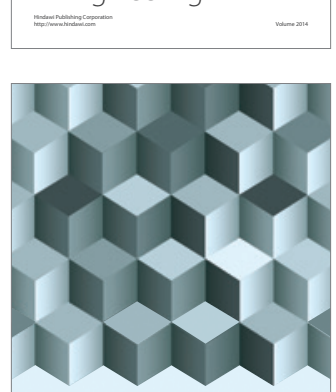

Journal of

Function Spaces
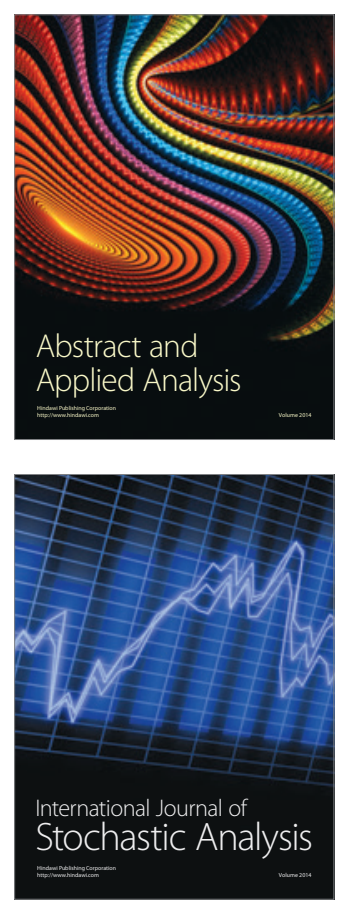

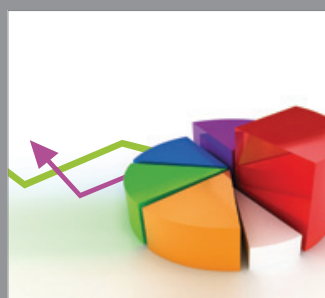

ournal of

Probability and Statistics

Promensencen
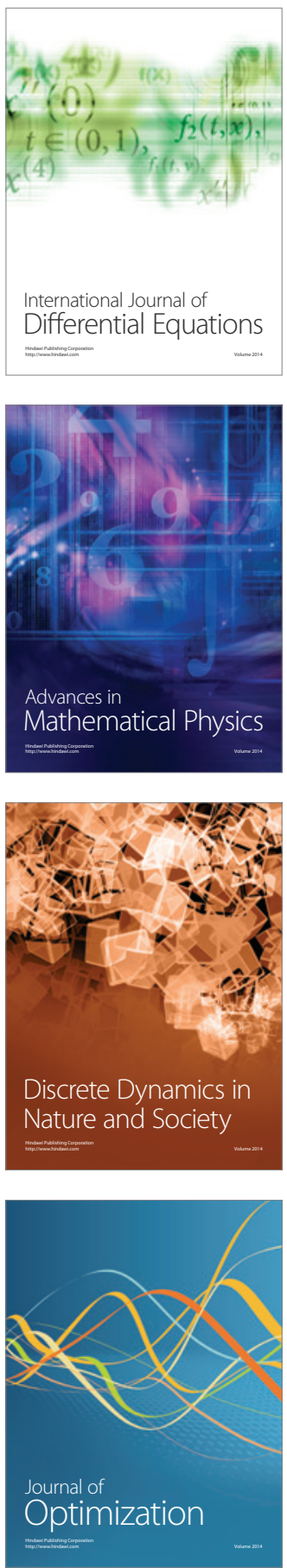